\title{
JOINT SPECTRA OF REPRESENTATIONS OF LIE ALGEBRAS BY COMPACT OPERATORS
}

\author{
ENRICO BOASSO \\ Departamento de Matemática, Facultad de Ciencias Exactas y Naturales, Universidad de Buenos Aires, \\ Ciudad Universitaria, Pabellón I, (1428) Buenos Aires, República Argentina, \\ e-mail:eboasso@dm.uba.ar
}

(Received 11 May, 2003; accepted 18 December, 2003)

\begin{abstract}
Given $X$ a complex Banach space, $L$ a complex nilpotent finite dimensional Lie algebra, and $\rho: L \rightarrow L(X)$, a representation of $L$ in $X$ such that $\rho(l) \in K(X)$ for all $l \in L$, the Taylor, the Słodkowski, the Fredholm, the split and the Fredholm split joint spectra of the representation $\rho$ are computed.

2000 Mathematics Subject Classification. Primary 47A13, 47A10. Secondary 17B15, $17 \mathrm{~B} 55$.
\end{abstract}

1. Introduction. Many of the well known joint spectra defined for commuting tuples of operators were extended to solvable Lie algebras of operators or, more generally, to representations of solvable Lie algebras in Banach spaces. For example, among such joint spectra, it is important to recall the Taylor, the Słodkowski, the Fredholm, the split and the Fredholm split joint spectra; see [2], [4], [5], [7], [8], [9], [10], [11], [12], [13] and [14].

In the work [3] nilpotent Lie algebras of linear transformations defined in finite dimensional Banach spaces were considered and the Słodkowski joint spectra, in particular the Taylor joint spectrum, of such an algebra were computed. In [1] this characterization was extended to representations of nilpotent Lie algebras by compact operators defined in Banach spaces, although only for the Taylor joint spectrum.

In this article representations of nilpotent Lie algebras by compact operators defined in a finite or infinite dimensional Banach space are considered and all the above mentioned joint spectra of such a representation are computed. These results extend the characterizations of [1] and [3].

The paper is organized as follows. In Section 2 some definitions and results needed for the present work are recalled, and in Section 3 the main characterizations are proved.

2. Joint spectra of representations of Lie algebras. In this section the definitions and the main properties of the Taylor, the Słodkowski, the Fredholm, the split and the Fredholm split joint spectra are reviewed; for a complete exposition see [2], [4], [5], [9], [11] and [12].

From now on $X$ denotes a complex Banach space, $L(X)$ the algebra of all bounded operators an $X, K(X)$ the compact operators an $X$, and $L(X, Y)$ the algebra of bounded

This research was supported by UBACYT. The author is a researcher of CONICET. 
linear maps from $X$ to $Y$, where $Y$ is another Banach space. If $X$ and $Y$ are two Banach spaces and $T \in L(X, Y)$, then the range and the null space of $T$ are denoted by $R(T)$ and $N(T)$ respectively. In addition, if $L$ is a complex solvable finite dimensional Lie algebra, then $\rho: L \rightarrow L(X)$ denotes a representation of $L$ in $X$.

If $X, L$ and $\rho$ are as above, it is possible to consider the Koszul complex of the representation $\rho$; that is $(X \otimes \wedge L, d(\rho))$, where $\wedge L$ denotes the exterior algebra of $L$ and $d_{p}(\rho): X \otimes \wedge^{p} L \rightarrow X \otimes \wedge^{p-1} L$ is the map defined by

$$
\begin{aligned}
& d_{p}(\rho)\left(x \otimes\left\langle l_{1} \wedge \ldots \wedge l_{p}\right\rangle\right) \\
& =\sum_{k=1}^{p}(-1)^{k+1} \rho\left(l_{k}\right)(x) \otimes\left\langle l_{1} \wedge \ldots \wedge \hat{l}_{k} \wedge \ldots \wedge l_{p}\right\rangle+\sum_{1 \leq i<j \leq p}(-1)^{i+j-1} x \\
& \quad \otimes\left\langle\left[l_{i}, l_{j}\right] \wedge l_{1} \wedge \ldots \wedge \hat{l}_{i} \wedge \ldots \wedge \hat{l}_{j} \wedge \ldots \wedge l_{p}\right\rangle,
\end{aligned}
$$

where ^ means deletion. If $\operatorname{dim} L=n$, then for $p$ such that $p \leq 0$ or $p \geq n+1, d_{p}(\rho)=0$.

In addition, if $f$ is a character of $L$, i.e. $f \in L^{*}$ and $f\left(L^{2}\right)=0$, where $L^{2}=\{[x, y]: x, y \in L\}$ and $[\cdot, \cdot]$ denotes the Lie bracket of $L$, then it is possible to consider the representation of $L$ in $X$ given by $\rho-f \equiv \rho-f \cdot I$, where $I$ is the identity map of $X$. If $H_{*}(X \otimes \wedge L, d(\rho-f))$ denotes the homology of the Koszul complex of the representation $\rho-f$, then it is possible to introduce the sets

$$
\sigma_{p}(\rho)=\left\{f \in L^{*}: f\left(L^{2}\right)=0, H_{p}(X \otimes \wedge L, d(\rho-f)) \neq 0\right\},
$$

and

$$
\sigma_{p, e}(\rho)=\left\{f \in L^{*}: f\left(L^{2}\right)=0, \operatorname{dim} H_{p}(X \otimes \wedge L, d(\rho-f))=\infty\right\} .
$$

Next follow the definitions of the Taylor, the Słodkowski, and the Fredholm joint spectra; see [2], [4], [5], [8], [9], [10], [11], [12], [13] and [14].

Definition 1. Let $X$ be a complex Banach space, $L$ a complex solvable finite dimensional Lie algebra, and $\rho: L \rightarrow L(X)$ a representation of $L$ in $X$. Then, the Taylor joint spectrum of $\rho$ is the set

$$
\sigma(\rho)=\cup_{p=0}^{n} \sigma_{p}(\rho)=\left\{f \in L^{*}: f\left(L^{2}\right)=0, H_{*}(X \otimes \wedge L, d(\rho-f)) \neq 0\right\} .
$$

In addition, the $k$-th $\delta$-Stodkowski joint spectrum of $\rho$ is the set

$$
\sigma_{\delta, k}(\rho)=\cup_{p=0}^{k} \sigma_{p}(\rho),
$$

and the $k$-th $\pi$-Stodkowski joint spectrum of $\rho$ is the set

$$
\sigma_{\pi, k}(\rho)=\cup_{p=n-k}^{n} \sigma_{p}(\rho) \cup\left\{f \in L^{*}: f\left(L^{2}\right)=0, R\left(d_{n-k}(\rho-f)\right) \text { is not closed }\right\},
$$

for $k=0, \ldots, n=\operatorname{dim} L$.

Observe that $\sigma_{\delta, n}(\rho)=\sigma_{\pi, n}(\rho)=\sigma(\rho)$.

On the other hand, the Fredholm or essential Taylor joint spectrum of $\rho$ is the set

$$
\sigma_{e}(\rho)=\cup_{p=0}^{n} \sigma_{p, e}(\rho) .
$$

In addition, the $k$-th Fredholm or essential $\delta$-Stodkowski joint spectra of $\rho$ is the set

$$
\sigma_{\delta, k, e}(\rho)=\cup_{p=0}^{k} \sigma_{p, e}(\rho),
$$


and the $k$-th Fredholm or essential $\pi$-Slodkowski joint spectrum of $\rho$ is the set

$$
\sigma_{\pi, k, e}(\rho)=\cup_{p=n-k}^{n} \sigma_{p, e}(\rho) \cup\left\{f \in L^{*}: f\left(L^{2}\right)=0, R\left(d_{n-k}(\rho-\delta)\right) \text { is not closed }\right\},
$$

for $k=0, \ldots, n$.

Observe that $\sigma_{e}(\rho)=\sigma_{\delta, n, e}(\rho)=\sigma_{\pi, n, e}(\rho)$.

In order to state the definition of the split and Fredholm split joint spectra, some preliminary facts are needed.

A finite complex of Banach spaces and bounded linear operators $(X, d)$ is a sequence

$$
0 \rightarrow X_{n} \stackrel{d_{n}}{\longrightarrow} X_{n-1} \rightarrow \ldots \rightarrow X_{1} \stackrel{d_{1}}{\longrightarrow} X_{0} \rightarrow 0,
$$

where $n \in \mathbb{N}, X_{p}$ are Banach spaces, and the maps $d_{p} \in L\left(X_{p}, X_{p-1}\right)$ are such that $d_{p-1} \circ d_{p}=0$, for $p=1, \ldots, n$.

Next, given a fixed integer $p, 0 \leq p \leq n$, the complex $(X, d)$ is called split (resp. Fredholm split) in degree $p$, if there are continous linear operators

$$
X_{p+1} \stackrel{h_{p}}{\longleftarrow} X_{p} \stackrel{h_{p-1}}{\longleftarrow} X_{p-1},
$$

such that $d_{p+1} h_{p}+h_{p-1} d_{p}=I_{p}$ (resp. $d_{p+1} h_{p}+h_{p-1} d_{p}=I_{p}-k_{p}$, for some $k_{p} \in K\left(X_{p}\right)$ ), where $I_{p}$ denotes the identity map of $X_{p}, p=0, \ldots, n$; see $[7,2]$.

In addition, if $L, X$ and $\rho$ are as above, and if $p$ is such that $0 \leq p \leq n$, then it is possible to introduce the sets

$$
s p_{p}(\rho)=\left\{f \in L^{*}: f\left(L^{2}\right)=0,(X \otimes \wedge L, d(\rho-f)) \text { is not split in degree } p\right\}
$$

and

$$
\begin{aligned}
s p_{p, e}(\rho)= & \left\{f \in L^{*}: f\left(L^{2}\right)=0,(X \otimes \wedge L, d(\rho-f))\right. \text { is not Fredholm split } \\
& \text { in degree } p\} .
\end{aligned}
$$

Next follow the definitions of the split and the Fredholm split joint spectra; see [4], [7] and [12].

Definition 2. Let $X$ be a complex Banach space, $L$ a complex solvable finite dimensional Lie algebra, and $\rho: L \rightarrow L(X)$ a representation of $L$ in $X$. Then, the split spectrum of $\rho$ is the set

$$
s p(\rho)=\cup_{p=0}^{n} s p_{p}(\rho) .
$$

In addition, the $k$-th $\delta$-split joint spectrum of $\rho$ is the set

$$
s p_{\delta, k}(\rho)=\cup_{p=0}^{k} s p_{p}(\rho),
$$

and the $k$-th $\pi$-split joint spectrum of $\rho$ is the set

$$
s p_{\pi, k}(\rho)=\cup_{p=n-k}^{n} s p_{p}(\rho),
$$

for $k=0, \ldots, n=\operatorname{dim} L$.

Observe that $s p_{\delta, n}(\rho)=s p_{\pi, n}(\rho)=s p(\rho)$.

On the other hand, the Fredholm or essential split spectrum of $\rho$ is the set

$$
s p_{e}(\rho)=\cup_{p=0}^{n} s p_{p, e}(\rho) .
$$


In addition, the $k$-th Fredholm or essential $\delta$-split joint spectrum of $\rho$ is the set

$$
s p_{\delta, k, e}(\rho)=\cup_{p=0}^{k} s p_{p, e}(\rho),
$$

and the $k$-th Fredholm or essential $\pi$-split joint spectrum of $\rho$ is the set

$$
s p_{\pi, k, e}(\rho)=\cup_{p=n-k}^{n} s p_{p, e}(\rho),
$$

for $k=0, \ldots, n$.

Observe that $s p_{\delta, n, e}(\rho)=s p_{\pi, n, e}(\rho)=s p_{e}(\rho)$.

It is clear that $\sigma_{\delta, k}(\rho) \subseteq s p_{\delta, k}(\rho), \sigma_{\pi, k}(\rho) \subseteq s p_{\pi, k}(\rho), \sigma(\rho) \subseteq s p(\rho)$, and $\sigma_{\delta, k, e}(\rho) \subseteq$ $s p_{\delta, k, e}(\rho), \sigma_{\pi, k, e}(\rho) \subseteq s p_{\pi, k, e}(\rho), \sigma_{e}(\rho) \subseteq s p_{e}(\rho)$. Moreover, if $X$ is a Hilbert space, then the inclusions above are equalities.

All the joint spectra considered above are defined for representations of complex solvable finite dimensional Lie algebras in complex Banach spaces and have the main spectral properties. That is, they are compact nonempty subsets of characters of the Lie algebra $L$ and they have the projection property for ideals; i.e. if $L$ is such a Lie algebra, $I$ is an ideal of $L, \sigma_{*}$ is one of the joint spectra above, and $\pi: L^{*} \rightarrow I^{*}$ denotes the restriction map from $L^{*}$ to $I^{*}$, then

$$
\pi\left(\sigma_{*}(L)\right)=\sigma_{*}(I) .
$$

For a complete exposition see the works [2], [4], [5], [9], [11] and [12].

In the following section representations of nilpotent Lie algebras by compact operators in finite or infinite dimensional Banach spaces will be considered and all the above mentioned joint spectra of such a representation will be computed. However, to this end, first it is necessary to recall a result from [4].

If $L, X$, and $\rho: L \rightarrow L(X)$ are as above, then it is possible to consider the representation

$$
L_{\rho}: L \rightarrow L(L(X)), l \rightarrow L_{\rho(l)},
$$

where $L_{\rho(l)}$ denotes the left multiplication operator associated to $\rho(l), l \in L$. In addition, since $L_{\rho(l)}(K(X)) \subseteq K(X)$, it is possible to consider the representation

$$
\tilde{L}_{\rho}: L \rightarrow L(C(X))
$$

where $C(X)=L(X) / K(X)$, and $\tilde{L}_{\rho}(l)$ is the quotient operator defined in $C(X)$ associated to $L_{\rho(l)}, l \in L$.

Similarly, if $L^{o p}$ is the Lie algebra which, as a vector space coincides with $L$ but whose Lie bracket is the opposite one, then it is possible to consider the representation

$$
R_{\rho}: L^{o p} \rightarrow L(L(X)), l \rightarrow R_{\rho(l)},
$$

where $R_{\rho(l)}$ denotes the right multiplication operator associated to $\rho(l), l \in L^{o p}$. Furthermore, since $R_{\rho(l)}(K(X)) \subseteq K(X)$, it is possible to consider the representation

$$
\tilde{R}_{\rho}: L^{o p} \rightarrow L(C(X)),
$$

where $\tilde{R}_{\rho}(l)$ is the quotient operator defined in $C(X)$ associated to $R_{\rho(l)}, l \in L^{o p}$. 
Now, if $L$ is a nilpotent Lie algebra, then according to $[4 ; 8]$ and $[\mathbf{1 2} ; 0.5 .8]$
(i) $s p_{\delta, k, e}(\rho)=\sigma_{\delta, k}\left(\tilde{L}_{\rho}\right)$,
(ii) $s p_{\pi, k, e}(\rho)=\sigma_{\delta, k}\left(\tilde{R}_{\rho}\right)$,
(iii) $\operatorname{sp}_{e}(\rho)=\sigma\left(\tilde{L}_{\rho}\right)=\sigma\left(\tilde{R}_{\rho}\right)$.

3. The main results. In this section, given a representation of a complex nilpotent finite dimensional Lie algebra by compact operators in a finite or infinite complex Banach space, the Taylor, the Słodkowski, the Fredholm, the split, and the Fredholm split joint spectra of such a representation are characterized. Since the example which follows Theorem 5 in [3] shows that for a solvable non-nilpotent Lie algebra of compact operators the characterization fails, only nilpotent Lie algebras are considered. In the first place the infinite dimensional case is studied.

In the following theorem the Taylor and the Słodkowski joint spectra are described.

THeOREM 1. Let $X$ be an infinite dimensional complex Banach space, L a complex nilpotent finite dimensional Lie algebra, and $\rho: L \rightarrow L(X)$ a representation of $L$ in $X$ such that $\rho(l) \in K(X)$ for each $l \in L$. Then, the sets $\sigma(\rho), \sigma_{\delta, k}(\rho) \cup\{0\}$, and $\sigma_{\pi, k}(\rho) \cup\{0\}$ coincide with the set

$\{0\} \cup\left\{f \in L^{*}: f\left(L^{2}\right)=0\right.$, such that there is $x \in X, x \neq 0$, with the property: $\rho(l)(x)=f(l) x, \forall l \in L\}$,

for $k=0, \ldots, n=\operatorname{dim} L$,

Proof. First of all recall that, according to $[1 ; 3.8],[1 ; 3.9]$ and $[9 ; 2.6]$,

$$
\begin{gathered}
\sigma(\rho)=\{0\} \cup\left\{f \in L^{*}: f\left(L^{2}\right)=0, \text { such that there is } x \in X, x \neq 0,\right. \\
\text { with the property } \rho(l)(x)=f(l) x, \forall l \in L\} .
\end{gathered}
$$

Now, since $L$ is a nilpotent Lie algebra, according to the above equality and Definition 1, $\sigma(\rho)=\{0\} \cup \sigma_{\pi, 0}(\rho)$. However, since $\sigma_{\pi, 0}(\rho) \cup\{0\} \subseteq \sigma_{\pi, k}(\rho) \cup\{0\} \subseteq \sigma(\rho)$, $0 \leq k \leq n$,

$$
\sigma(\rho)=\sigma_{\pi, k} \cup\{0\}=\sigma_{\pi, 0}(\rho) \cup\{0\} .
$$

On the other hand, if the adjoint representation of $\rho$ is considered, i.e. the representation $\rho^{*}: L^{o p} \rightarrow L\left(X^{\prime}\right), \rho^{*}(l)=(\rho(l))^{*}$, where $X^{\prime}$ denotes the dual space of $X$ then, since $L^{o p}$ is a nilpotent Lie algebra, according to [12; 0.5.8] and [12; 2.11.4],

$$
\{0\} \cup \sigma_{\delta, k}(\rho)=\{0\} \cup \sigma_{\pi, k}\left(\rho^{*}\right)=\{0\} \cup \sigma_{\pi, 0}\left(\rho^{*}\right)=\sigma\left(\rho^{*}\right)=\sigma(\rho) .
$$

In the following theorem the Fredholm joint spectra are computed.

THEOREM 2. Let $X$ be an infinite dimensional complex Banach space, L a complex nilpotent finite dimensional Lie algebra, and $\rho: L \rightarrow L(X)$ a representation of $L$ in $X$ such that $\rho(l) \in K(X)$ for each $l \in L$. Then

$$
\sigma_{e}(\rho)=\sigma_{\delta, k, e}(\rho)=\sigma_{\pi, k, e}(\rho)=\{0\},
$$

for $k=0, \ldots, n=\operatorname{dim} L$. 
Proof. The proof is based on an induction argument on the dimension of the algebra. If $\operatorname{dim} L=1$, then consider $l \in L$ such that $\langle l\rangle=L$. Now, since $\rho(l)$ is a compact operator, $\sigma_{e}(\rho(l))=\{0\}$. However, since $\operatorname{dim} L=1$, according to Definition $1, \sigma_{e}(\rho)=\{0\}$. Now, since $\sigma_{\delta, k, e}(\rho)$ and $\sigma_{\pi, k, e}(\rho)$ are nonempty subsets of $\sigma_{e}(\rho)$,

$$
\sigma_{e}(\rho)=\sigma_{\delta, k, e}(\rho)=\sigma_{\pi, k, e}(\rho)=\{0\},
$$

$0 \leq k \leq 1=\operatorname{dim} L$

Now suppose that for every nilpotent Lie algebra of dimension less than $n$ and for every representation of the algebra by compact operators in an infinite dimensional Banach space $X$ we have

$$
\sigma_{e}(\rho)=\sigma_{\delta, k, e}(\rho)=\sigma_{\pi, k, e}(\rho)=\{0\}
$$

Next, if $L$ is a nilpotent Lie algebra of dimension $n$ then, according to [6; 5.1], there is a Jordan-Hölder sequence of ideals $\left(L_{i}\right)_{0 \leq i \leq n}$ such that

(i) $L_{0}=0$, and $L_{n}=L$,

(ii) $L_{i} \subseteq L_{i+1}, 0 \leq i \leq n-1$,

(iii) $\left[L_{i}, L_{j}\right] \subseteq L_{i-1}$, for $i<j$.

It is easy to prove that $L^{2} \subseteq L_{n-2}$. Then, it is possible to consider the ideals $I_{1}=L_{n-1}$ and $I_{2}=L_{n-2} \oplus\langle x\rangle$, where $x \in L$ is such that $L_{n-1} \oplus\langle x\rangle=L$.

On the other hand, if the representations $\rho_{1}=\rho \mid I_{1}: I_{1} \rightarrow L(X)$ and $\rho_{2}=$ $\rho \mid I_{2}: I_{2} \rightarrow L(X)$ are considered, then, by the assumption under consideration,

$$
\sigma_{e}\left(\rho_{1}\right)=\sigma_{\delta, k, e}\left(\rho_{1}\right)=\sigma_{\pi, k, e}\left(\rho_{1}\right)=\{0\},
$$

and

$$
\sigma_{e}\left(\rho_{2}\right)=\sigma_{\delta, k, e}\left(\rho_{2}\right)=\sigma_{\pi, k, e}\left(\rho_{2}\right)=\{0\}
$$

However, according to the projection property of the Fredholm joint spectra, $[4 ; 3.2]$ and $[4 ; 3.5]$,

$$
\sigma_{e}(\rho)=\sigma_{\delta, k, e}(\rho)=\sigma_{\pi, k, e}(\rho)=\{0\},
$$

for $k=0, \ldots, n=\operatorname{dim} L$.

In the following theorem the split and the Fredholm split joint spectra are computed.

THeOREM 3. Let $X$ be an infinite dimensional complex Banach space, L a complex nilpotent finite dimensional Lie algebra, and $\rho: L \rightarrow L(X)$ a representation of $L$ in $X$ such that $\rho(l) \in K(X)$ for each $l \in L$. Then, $\sigma(\rho)=s p(\rho), \sigma_{\delta, k}(\rho)=s p_{\delta, k}(\rho)$ and $\sigma_{\pi, k}(\rho)=s p_{\pi, k}(\rho)$, where $k=0, \ldots, n=\operatorname{dim} L$.

In particular, the sets $\sigma(\rho), \sigma_{\delta, k}(\rho) \cup\{0\}, \sigma_{\pi, k}(\rho) \cup\{0\}, s p(\rho), s p_{\delta, k}(\rho) \cup\{0\}$ and $s p_{\pi, k}(\rho) \cup\{0\}$ coincide with the set

$\{0\} \cup\left\{f \in L^{*}: f\left(L^{2}\right)=0\right.$, such that there is $x \in X, x \neq 0$, with the property $\rho(l)(x)=f(l) x, \forall l \in L\}$. 
In addition,

$$
s p_{e}(\rho)=s p_{\delta, k, e}(\rho)=s p_{\pi, k, e}(\rho)=\{0\},
$$

where $k=0, \ldots, n=\operatorname{dim} L$.

In particular, all the Fredholm and Fredholm split joint spectra coincide with the set $\{0\}$.

Proof. First of all, since $\rho(L) \subseteq K(X), \tilde{L}_{\rho}=0$ and $\tilde{R}_{\rho}=0$. Thus, since $L$ is a nilpotent Lie algebra, according to $[4 ; 8]$ and $[12 ; 0.5 .8]$, we have

(i) $s p_{\delta, k, e}(\rho)=\sigma_{\delta, k}\left(\tilde{L}_{\rho}\right)=\{0\}$,

(ii) $s p_{\pi, k, e}(\rho)=\sigma_{\delta, k}\left(\tilde{R}_{\rho}\right)=\{0\}$,

(iii) $\operatorname{sp}_{e}(\rho)=\sigma\left(\tilde{L}_{\rho}\right)=\sigma\left(\tilde{R}_{\rho}\right)=\{0\}$.

Next, in order to prove the first assertion of the Theorem, observe that, according to $[7 ; 2.7]$,

(i) $\operatorname{sp}(\rho)=\sigma(\rho) \cup \mathcal{C}, s p_{e}(\rho)=\sigma_{e}(\rho) \cup \tilde{\mathcal{C}}$,

(ii) $s p_{\delta, k}(\rho)=\sigma_{\delta, k}(\rho) \cup \mathcal{A}_{k}, s p_{\delta, k, e}(\rho)=\sigma_{\delta, k, e}(\rho) \cup \tilde{\mathcal{A}}_{k}$,

(iii) $s p_{\pi, k}(\rho)=\sigma_{\pi, k}(\rho) \cup \mathcal{B}_{k}, s p_{\pi, k, e}(\rho)=\sigma_{\pi, k, e}(\rho) \cup \tilde{\mathcal{B}}_{k}$, where $k=0, \ldots, n$ and

(iv) $\mathcal{A}_{k}=\left\{f \in L^{*}: f\left(L^{2}\right)=0, f \notin \sigma_{\delta, k}(\rho)\right.$, and there is $p, p=1, \ldots, k+1$, such that $N\left(d_{p}(\rho-f)\right)$ is not complemented in $\left.X \otimes \wedge^{p} L\right\}$,

(v) $\tilde{\mathcal{A}}_{k}=\left\{f \in L^{*}: f\left(L^{2}\right)=0, f \notin \sigma_{\delta, k, e}(\rho)\right.$, and there is $p, p=1, \ldots, k+1$, such that $N\left(d_{p}(\rho-f)\right)$ is not complemented in $X \otimes \wedge^{p} L$,

(vi) $\mathcal{B}_{k}=\left\{f \in L^{*}: f\left(L^{2}\right)=0, f \notin \sigma_{\pi, k}(\rho)\right.$, and there is $p, p=n, \ldots, n-k$, such that $R\left(d_{p}(\rho-f)\right)$ is not complemented in $\left.X \otimes \wedge^{p-1} L\right\}$,

(vii) $\tilde{\mathcal{B}}_{k}=\left\{f \in L^{*}: f\left(L^{2}\right)=0, f \notin \sigma_{\pi, k, e}(\rho)\right.$, and there is $p, p=n, \ldots, n-$ $k$, such that $R\left(d_{p}(\rho-f)\right)$ is not complemented in $\left.X \otimes \wedge^{p-1} L\right\}$,

(viii) $\mathcal{C}=\mathcal{A}_{n}=\mathcal{B}_{n}, \tilde{\mathcal{C}}=\tilde{\mathcal{A}}_{n}=\tilde{\mathcal{B}}_{n}$.

In addition, $\mathcal{A}_{k} \subseteq \tilde{\mathcal{A}}_{k}$, and $\mathcal{B}_{k} \subseteq \tilde{\mathcal{B}}_{k}$, for $k=0, \ldots, n$.

However, since $\sigma_{\delta, k, e}(\rho) \cap \tilde{\mathcal{A}}_{k}=\emptyset$ and $\sigma_{\pi, k, e}(\rho) \cap \tilde{\mathcal{B}}_{k}=\emptyset$, according to Theorem 2 and to the second part of the Theorem, which have already been proved, $\tilde{\mathcal{A}}_{k}=\emptyset$ and $\tilde{\mathcal{B}}_{k}=\emptyset$. In particular, $\mathcal{A}_{k}=\emptyset$ and $\mathcal{B}_{k}=\emptyset$, which proves the first assertion of the Theorem.

Next, representations of nilpotent Lie algebras in finite dimensional Banach spaces are considered. Observe that in this case the spectra to be studied are the Taylor and the Słodkowski joint spectra.

THEOREM 4. Let $X$ be a complex finite dimensional Banach space, L a complex nilpotent finite dimensional Lie algebra, and $\rho: L \rightarrow L(X)$ a representation of $L$ in $X$. Then

$$
\begin{aligned}
\sigma(\rho) & =\sigma_{\delta, k}(\rho)=\sigma_{\pi, k}(\rho)=\left\{f \in L^{*}: f\left(L^{2}\right)=0, \text { such that there is } x \in X,\right. \\
x & \neq 0, \text { with the property } \rho(l) x=f(l) x, \forall l \in L\} .
\end{aligned}
$$

Proof. Since $L$ is a nilpotent Lie algebra, according to $[3 ; 4]$ and $[9 ; 2.6]$,

$$
\begin{aligned}
\sigma(\rho)= & \left\{f \in L^{*}: f\left(L^{2}\right)=0, \text { such that there is } x \in X, x \neq 0,\right. \text { with the } \\
& \text { property } \rho(l) x=f(l) x, \forall l \in L\} .
\end{aligned}
$$


Therefore, since $L$ is a nilpotent Lie algebra, $\sigma(\rho)=\sigma_{\pi, 0}(\rho)$. Finally, in order to finish the proof, it is possible to use an argument similar to that developed in Theorem 1.

\section{REFERENCES}

1. D. Beltita, Spectrum for a solvable Lie algebra of operators, Studia Math. 135 (1999), $163-178$

2. E. Boasso, Dual properties and joint spectra for solvable Lie algebras of operators, J. Operator Theory 33 (1995), 105-116.

3. E. Boasso, Joint spectra and nilpotent Lie algebras of linear transformations, Linear Algebra Appl. 263 (1997), 49-62.

4. E. Boasso, Joint spectra of the tensor product representation of the direct sum of two Lie algebras, Dissertationes Math. 416 (2003).

5. E. Boasso and A. Larotonda, A spectral theory for solvable Lie algebras of operators, Pacific J. Math. 158 (1993), 15-22.

6. N. Bourbaki, Éléments de Mathématique, Groupes et Algèbres de Lie, Chapitre I, Algèbres de Lie, Fasc. XXVI (Hermann, Paris 1960).

7. J. Eschmeier, Analytic spectral mapping theorems for joint spectra, in Operators in indefinite metric spaces, scattering theory and other topics (Bucharest, 1985), Oper. Theory Adv. Appl. 24 (1987), 167-181.

8. A. S. Fainshtein, Joint essential spectrum of a family of linear operators, Funct. Anal. Appl. 14 (1980), 152-153.

9. A. S. Fainshtein, Taylor joint spectrum for families of operators generating nilpotent Lie algebras, J. Operator Theory 29 (1993), 3-27.

10. V. Müller, The Słodkowski spectra and higher Shilov Boundaries, Studia Math. 105 (1993), 69-75.

11. C. Ott, A note on a paper of E. Boasso and A. Larotonda, Pacific J. Math. 173 (1996), $173-179$

12. C. Ott, Gemeinsame Spektren auflösbarer Operator-Liealgebren, Dissertation, Kiel, 1997 (http://analysis.math.uni-kiel.de/wrobel/).

13. Z. Słodkowski, An infinite family of joint spectra, Studia Math. LXI (1973), 239-255.

14. J. L. Taylor, A joint spectrum for several commuting operators, J. Functional Analysis 6 (1970), 172-191. 